\title{
Gravitational Lensing, Dark Matter and the Optical Gravitational Lens Experiment
}

\author{
J. Surdej ${ }^{1 *}$, J.-F. Claeskens ${ }^{*}$, C. Delacroix ${ }^{2 *}$, T. Sadibekova ${ }^{3 *}$, P. Bartczak ${ }^{4 *}$, \\ P.H.I. Coleman ${ }^{\dagger}$, A. Magette ${ }^{5 *}$, J. Poels ${ }^{*}$, D. Ricci ${ }^{*}$ and D. Sluse ${ }^{* *, \sharp}$ \\ *Université de Liège, Département d'Astrophysique, Géophysique et Océanographie, Bât. B5C, \\ Sart Tilman, B-4000 LIEGE 1, Belgium \\ $\dagger$ Institute for Astronomy, University of Hawai'i, 2680 Woodlawn Drive, Honolulu, HI 96822, USA \\ ** Laboratoire d'Astrophysique, Ecole Polytechnique Fédérale de Lausanne (EPFL) Observatoire, \\ 1290 Versoix, Switzerland \\ E-mail:Surdej@astro.ulg.ac.be,Claeskens@astro.ulg.ac.be,CDelacroix@ulg.ac.be, \\ Sadibekova@astro.ulg.ac.be,Bartczak@astro.ulg.ac.be,Gruff@ifa.hawaii.edu, \\ Magette@astro.ulg.ac.be,Poels@astro.ulg.ac.be,Ricci@astro.ulg.ac.be, \\ Dominique.Sluse@epfl.ch
}

\begin{abstract}
After briefly reviewing the history of gravitational lensing, we recall the basic principles of the theory.

We then describe and use a simple optical gravitational lens experiment which has the virtue of accounting for all types of image configurations observed so far among the presently known gravitational lens systems.

Finally, we briefly present the $4 \mathrm{~m}$ International Liquid Mirror Telescope project in the context of a photometric monitoring of multiply imaged quasars.
\end{abstract}

Keywords: Gravitational lensing, Multiply imaged quasars, Dark matter, Liquid Mirror Telescope, Optical gravitational lens experiment

PACS: 98.62.Sb, 42.79.Bh, 95.55.Cs, 98.54.-h

\section{INTRODUCTION}

A mirage is an optical illusion... whose cause is real! But what is really a mirage? In order to properly answer this question let us consider an observer $O$ looking at a distant source $S$. Under normal circumstances (straight propagation of light rays), the observer $O$ sees from the distant source $S$ a single image $I$, located exactly along the direction of the incoming light ray. In this case, the real position of the source $S$ coincides with that of the image $I$ (cf. Fig. 1A). A mirage is formed when the incoming light rays do not propagate along straight lines. The image $I$ may then be somewhat displaced with respect to the real source $S$ position (cf. Fig. 1B). Light may also propagate from a

1 also, Directeur de Recherche honoraire du FNRS

2 boursier FRIA

3 post-doc, département AGO

4 post-doc FNRS

5 boursier FRIA 
(A)

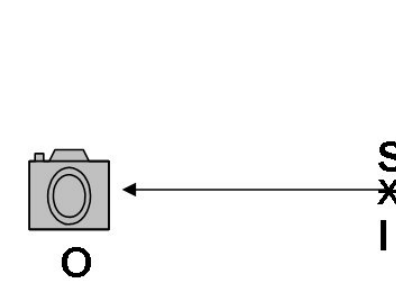

(B)

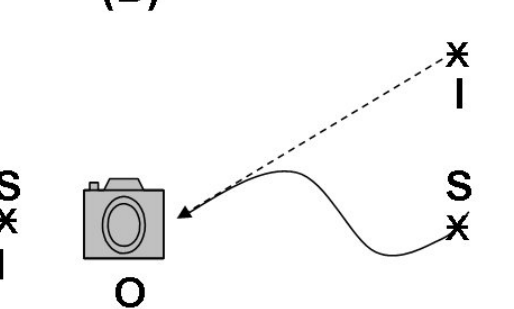

(C)

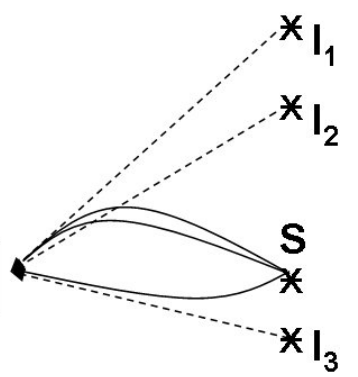

FIGURE 1. Different types of light ray propagation between a distant source $S$ and an observer $O$. The observer $O$ either sees (A) a single, (B) a slightly displaced or (C) multiple image(s) $I$ of the background source $S$.

distant source $S$ along different trajectories, their propagation times corresponding to extrema (Fermat Principle). These lead to the formation of multiple images $I_{i}$ of a single source $S$ (see Fig. $1 \mathrm{C}$ ) as seen by the observer $O$. Note that the observer $O$ always sees the corresponding image $I_{i}$ along the direction which is tangent to the incoming light ray. The cause of the bending of the light rays can be manifold. For instance, the presence of a temperature gradient above the ground may induce deflections of light rays during their propagation. Indeed, a temperature difference causes a variation in the density of air, which means a variation in the refraction index and there, the observer can occasionally witness an atmospheric mirage.

The deviation of the light rays may also be caused by an ad-hoc optical lens, alike in the optical gravitational lens experiment presented hereafter. Gravitational fields can also bend light rays as it was predicted by Einstein during the elaboration of the theory of General Relativity (1912; see quote in [7]). A gravitational lens mirage occurs when light rays are curved by the gravitational field of a massive object located between a distant source and an observer. This type of mirage is also called multiple gravitationally lensed images or still a gravitational lens system.

Unlike most astrophysical discoveries made during the past century, the physics of gravitational lensing was understood well before the first example of a multiply imaged source was found (see Einstein 1912 quoted in [7]). The existence of multiply imaged, distant sources had been predicted by Zwicky in 1937 [11]... although the first case of a doubly imaged quasar was only reported in 1979 [10]. We refer the reader to [3] for a more detailed account on the history of gravitational lensing.

\section{GRAVITATIONAL LENSING AND THE OPTICAL LENS EXPERIMENT}

\subsection{Gravitational lensing}

Einstein [4] has shown that a massive object, irrespective of its content in luminous and/or dark matter, curves the spacetime in its vicinity and that any particle, massive or not (cf. the photons), will move along the geodesics of this curved space. He found that 
(A): Gravitational lensing

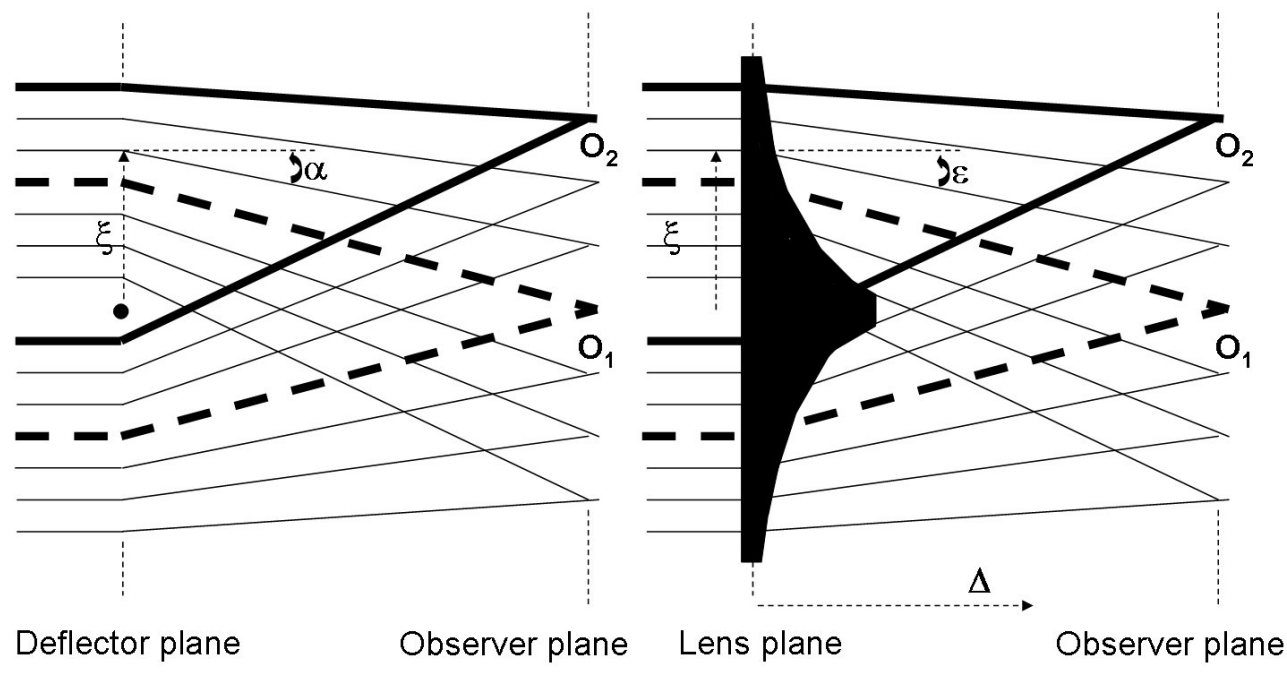

FIGURE 2. Gravitational lens deflection of a beam of parallel light rays coming from a very distant source located at left in (A). The light rays propagate in the plane containing the source, the deflector and the observer. In this case, the deflector is supposed to be a massive compact object represented by the black dot, and the light deflection angle $\alpha$ is given by Eq. (1) for $\mathbf{M}(\xi)=\mathbf{M}=\mathbf{C t e}$. As shown in this figure, the observer (cf. $\mathrm{O}_{2}$ ) always sees two lensed images of the distant source, except in the perfect symmetric case for which the observer $O_{1}$ sees a complete Einstein ring. At right (B) is shown the shape of an optical lens that accounts for the same deviation of the beam of parallel light rays (see [6]for a complete description). The optical lens looks like the foot of an inclined $\left(\right.$ at $\left.90^{\circ}\right)$ glass of wine. The observers in $O_{1}$ and $\mathrm{O}_{2}$ in (B) see similar mirages as the corresponding observers in (A).

a light ray passing at a distance (impact parameter) $\xi$ from an object characterized by an axially symmetric mass distribution $\mathbf{M}(\xi)$ (see Fig. 2A) will undergo a total deflection angle $\alpha$, expressed in radian, by means of the relation

$$
\alpha=\frac{4 G \mathbf{M}(\xi)}{c^{2} \xi},
$$

where $G$ stands for the gravitational constant and c for the velocity of light. Adopting a given mass distribution (e.g. a constant mass $\mathbf{M}(\xi)=\mathbf{M}$ in order to characterize a pointlike object, another mass distribution $\mathbf{M}(\xi)$ for the disk of a spiral galaxy, etc.), it is then trivial to construct an optical lens that deflects light rays accordingly (cf. Fig. 2B), thus enabling us to study very simply in the laboratory the lensing properties of black holes, stars, quasars, galaxies, etc. as they exist in the Universe. Before discussing the optical gravitational lens experiment, we wish to state that a sufficient condition for a gravitational lens to produce multiple images of a background source is simply that its surface mass density $\Sigma(\xi)$ exceeds the critical surface density $\Sigma_{c}$, which only depends on the relative angular distances $D_{o d}, D_{o s}$ and $D_{d s}$, between the observer $(o)$, the deflector 


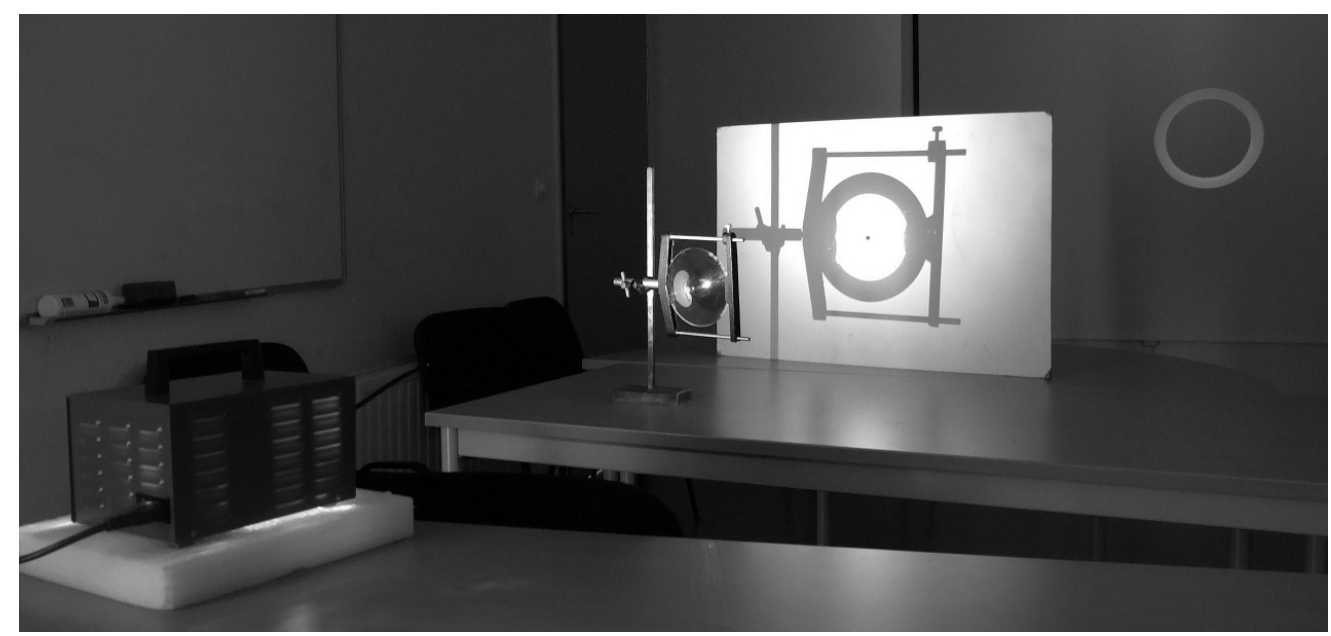

FIGURE 3. Setup of the gravitational lens experiment (see text). The optical gravitational lens (black hole type deflector) has been set perpendicularly to, and centred on the line joining the pinhole and the light source (see text).

$(d)$ and the source $(s)$ (see [6], [2]):

$$
\Sigma_{c}=\frac{c^{2}}{4 \pi G} \frac{D_{o s}}{D_{o d} D_{d s}} .
$$

It is also very easy to show that in the case of a perfect alignment between a source, an axially symmetric deflector and an observer (cf. $O_{1}$ in Fig. 2A), the latter will see the background source as a ring of light (the so-called Einstein ring) which angular radius $\Theta_{E}$ is proportional to the square root of the mass of the deflector (cf. [6]):

$$
\Theta_{E}=\sqrt{\frac{4 G \mathbf{M}(\xi)}{c^{2}} \frac{D_{d s}}{D_{o d} D_{o s}}} .
$$

\subsection{The optical gravitational lens experiment}

For didactical purposes, it is very useful to construct and use optical lenses that mimic the deflection of light rays as derived in Eq. (1) for the case of axially symmetric gravitational lenses. Such optical lenses should of course be rotationally symmetric, flat on one side (for simplicity) and have, on the other side, a surface determined in such a way that rays characterized by an impact parameter $\xi$ gets deflected by the angle $\varepsilon(\xi)=\alpha(\xi)$ (see Figs. 2A and 2B). The angle $\varepsilon(\xi)$ is of course directly related to the refraction properties of the lens (shape of its surface and value of the refraction index). Optical lenses simulating the light deflection properties due to a point mass (cf. a black hole, a star, a planet,... ) have been manufactured by us and correspond to the shape of the foot of a wine glass, as illustrated in Fig. $2 \mathrm{~B}$. It can be shown that the thickness 
$\Delta(\xi)$ of the lens as a function of the impact parameter $\xi$ is merely given by the equation (see [6])

$$
\frac{\mathrm{d} \Delta}{\mathrm{d} \xi}=\frac{-4 G M(\xi)}{(n-1) c^{2} \xi}
$$

where $n$ represents the refractive index of the optical lens. Note the values $n=1.49$ and $\mathbf{M}(\xi)=\mathbf{M}=\mathbf{C t e}$ for the case of the Plexiglas lens simulating the deflecting properties of a compact mass deflector that has been used during the Heidelberg-Liège-Paris-Wroclaw Joint Meeting held in Sol Cress (Spa, Belgium) on 6 March 2008. By integrating Eq. (4) and for a value of the mass $M$ corresponding to a black hole having approximately a mass of 8 times that of the Earth, we easily derive that $\Delta(\xi)=9 \ln (65 / \xi)+3$, where $\Delta$ and $\xi$ are both expressed in millimeters. The diameter of the optical lens shown in Fig. 3 is thus $13 \mathrm{~cm}$. Such lenses can be purchased via the Faculty of Sciences, Liège University, at a non profit cost of $40 €$ (see the link http://www2.ulg.ac.be/sciences/lentille/ index.htm).

In order to simulate the formation of lensed images for the case of the point mass deflector, we have used the optical setup shown in Fig. 3 .

A compact light source (representing e.g. a distant quasar) is located on the left hand side. Then comes the optical lens, that deviates in our case the light rays like a compact mass object (cf. a black hole). On the other side of the lens, we find a white screen with a very small hole at the center (the pinhole lens). Further beyond, there is a large screen on which is (are) projected the lensed image(s) of the source (an Einstein ring in this case) as it would be seen if our eye were located at the position of the pinhole. In the absence of an intervening deflector, the large background screen appears to be uniformly illuminated, and the observer only sees the single image of a distant quasar (see Fig 4). When setting the black hole lens perpendicularly to the rays coming from the distant source (see Fig. 3), neither the pinhole screen nor the background one are any longer uniformly illuminated. The black hole has redistributed the light in such a way that there is a maximum of light concentration along a bright focal line connecting the source and the lens. There is no single focus, the black hole acts as an imperfect lens... somewhat like a lens affected by spherical aberration. The intersection of this bright focal line with the pinhole screen is seen as a bright spot in Fig. 3. Thus, the maximum amplification is obtained when the pinhole (the observer eye) is very precisely set on the optical axis, corresponding to the formation of an Einstein ring. Farther away from the optical axis and in a plane perpendicular to it, the light gets dimmer; the distribution of light does in fact correspond there to convergence points due to two light rays being deflected by the lens, and the total amplification of the images tends towards unity with increasing distance from the axis of symmetry. We may easily observe that if the pinhole lens is set somewhat aside from the bright spot (see Fig. 5, at right), the Einstein ring breaks in two lensed images with a typical angular separation equal to the diameter of the Einstein ring. As we may expect, symmetric lenses seldom occur in nature; usually the main lens itself is non-symmetric, or some non-symmetric disturbances are induced by the presence of neighboring masses. In our gravitational lens experiment, the effects of a typical non-symmetric gravitational lens may be simulated by simply tilting the optical lens with respect to the line connecting the source and the lens. The bright focal line along the optical axis that existed in the symmetric configuration then becomes a two- 


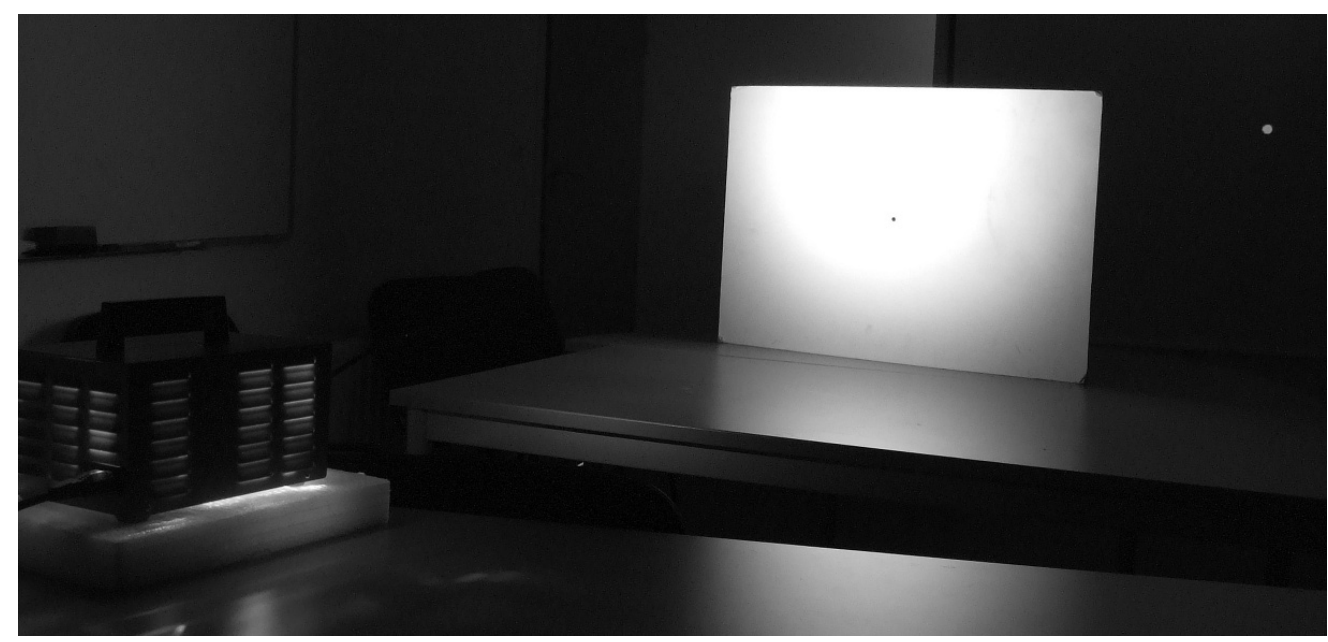

FIGURE 4. If there is no gravitational lens along the light rays, the pinhole camera projects on the background screen a single image of the light source.
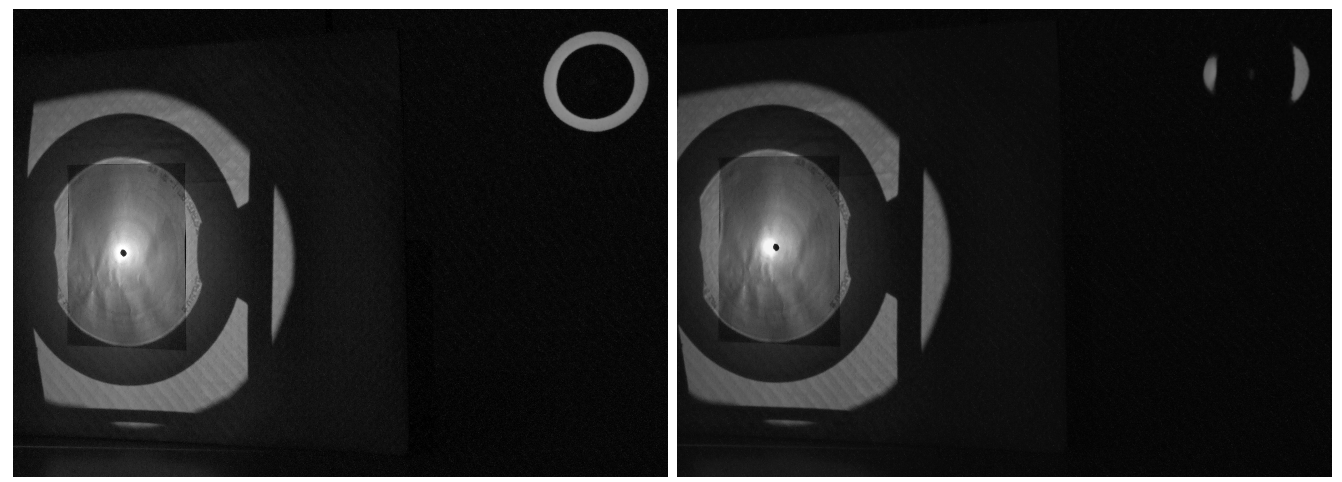

FIGURE 5. Left: The pinhole is on the symmetry axis, showing the Einstein ring on the background screen. Right: As the pinhole is set slightly away from the symmetry axis, the Einstein ring breaks up in two lensed images (see text).

dimensional envelope, called a caustic in optics. A section of this caustic is visible as a closed curve having a diamond shape (made of four folds and four cusps) in the pinhole plane (see Fig. 6).

The word "caustic" in gravitational lensing always refers to this section of the optical two-dimensional caustic (in the symmetric case, the caustic degenerates into a single spot, Fig. 3). As a result, the Einstein ring that was observed in the symmetric case is now split into four lensed images. Depending on where exactly the pinhole lens is located with respect to the caustic, different generic configurations of lensed images are produced (see Fig. 6). These are also illustrated in Fig. 7 and compared with typical observations of multiply imaged extragalactic sources. All image configurations observed for gravitational lens systems in the Universe can actually be reproduced with this straightforward gravitational lens experiment. 

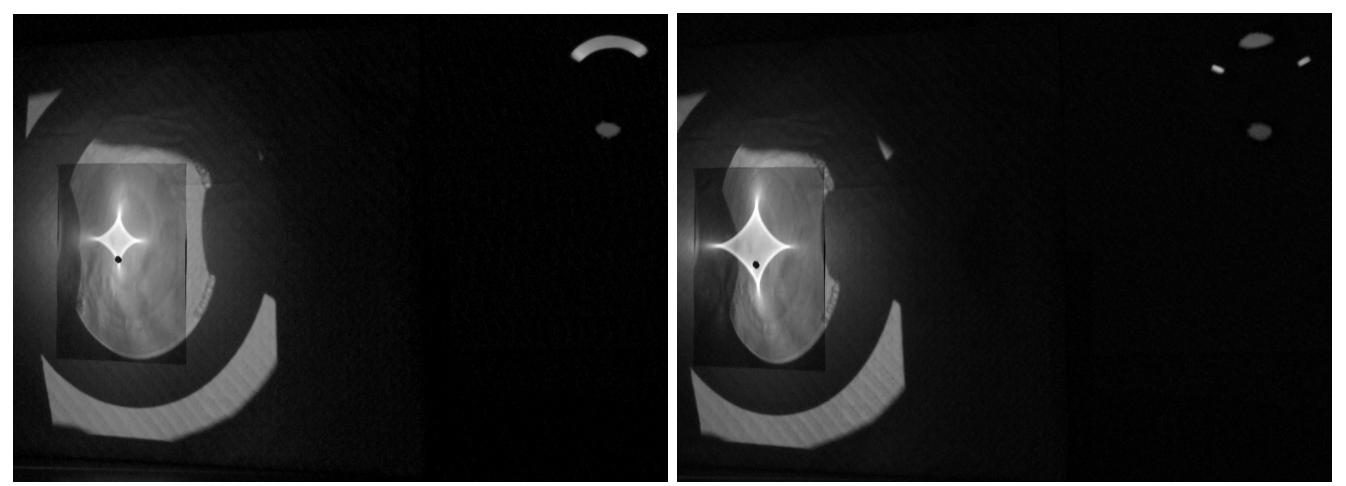

FIGURE 6. The optical gravitational lens experiment corresponding to the case of a black hole deflector that has been slightly tilted around its vertical axis. The bright (pseudo focal) line along the optical axis which existed in the symmetric configuration (cf. Figs. 3 and 5 has changed into a two dimensional caustic surface, a section of which is seen as a diamond shaped caustic (made of four folds and four cusps) in the pinhole plane. Seen on the background screen, the Einstein ring is now split into three merging lensed images plus a very faint one on the other side (left picture, with pinhole on the cusp) or in four distinct lensed images (right picture, with pinhole inside the caustic).

\section{THE INTERNATIONAL LIQUID MIRROR TELESCOPE PROJECT}

The International Liquid Mirror Telescope (ILMT) consists of a 4m zenithal telescope which is being presently constructed in Liège (see Fig. 8) under the leadership of the AEOS (J. Surdej, project scientist), GAPHE (J.-P. Swings, co-project manager) and Hololab (S. Habraken, co-project manager) teams (see a recent description in [9]). The $4 \mathrm{~m}$ ILMT will be erected in Devasthal (India) and will be entirely dedicated to the direct imaging of a narrow strip of sky $\left(\approx 30^{\prime}\right)$ at a constant declination near $+29^{\circ} 23^{\prime}$. The images will be acquired in the Time Delay Integration (TDI) mode and the telescope should become operational at the beginning of 2009 .

Given the small number $(\approx 100)$ of known multiply imaged quasars, almost randomly distributed over the sky, the probability to observe even only one of these within the $\approx 30^{\prime}$ zenithal field of view of the ILMT is virtually zero. Therefore, the observational strategy consists in first surveying a sky area as deep and as wide as possible for interesting targets, alike quasars and multiply imaged quasar candidates, on the basis of variability criteria. Surdej and Claeskens [8] have estimated that at least 50 new gravitationally lensed QSOs should be identified. Although this number sounds modest, the unique advantage of the ILMT consists in getting each night photometric measurementsfor the visible systems, down to the limiting magnitude $i^{\prime}=22.5$. This monitoring will make possible to construct lightcurves and derive the so-called time-delays between the flux variations of the lensed images. Time delays represent a crucial information to derive the value of the Hubble constant $H_{0}$ from gravitational lensing, if the mass distribution in the lens is sufficiently well modeled [5], or conversely, to constrain the mass distribution (luminous and dark matter) in the lensing galaxies adopting the value of $H_{0}$ as derived from independent techniques (cf. type Ia supernovae, WMAP observations). The Liège astronomers will lead the "gravitational lensing" project and a group of Canadian astro- 
nomers - also members of the 4m ILMT consortium - will collaborate with them. In addition, the latter plans to lead the project of monitoring type Ia supernovae that will be detected with the 4m ILMT (more than 1000 supernovae ought to be discovered in one year; [1]). Astronomers from the Belgium Royal Observatory and the Observatory of Nainithal (India) are also associated to the ILMT project.

\section{ACKNOWLEDGMENTS}

The ILMT project is supported by contract Action de Recherche Concertée "Space and Ground-based Imaging in Astrophysics" (2007-2011, Liège University), by the "Communauté Française" and the "Région Wallonne" de Belgique, by the "Fonds National de la Recherche Scientifique" and the "Liège University", Belgium.

\section{REFERENCES}

1. E. F. Borra. Astronomical Research with Liquid Mirror Telescopes. ArXiv Astrophysics e-prints, February 2001.

2. J.-F. Claeskens and J. Surdej. Gravitational lensing in quasar samples. Astronomy and Astrophysics Reviews, 10:263-311, 2002. doi: 10.1007/s001590000010.

3. J.-F. Claeskens and J. Surdej. Book Review: The century of space sciences / Two volumes, Johan A. M. Bleeker, Johannes Geiss, Martin C. E. Huber (eds.), Kluwer Academic, ISBN 0723-7196-8. Astronomy Now (ISSN 0951-9726), Vol. 17, No. 8, p. 20 (2003), 17:441-469, August 2003.

4. A. Einstein. Die Feldgleichungen der Gravitation (The Field Equations of Gravitation). Koniglich Preussische Akademie der Wissenschaften, pages 844-847, 1915.

5. S. Refsdal. On the possibility of determining Hubble's parameter and the masses of galaxies from the gravitational lens effect. Monthly Notices of the RAS, 128:307-+, 1964.

6. S. Refsdal and J. Surdej. Gravitational lenses. Reports of Progress in Physics, 57:117-185, 1994.

7. J. Renn, T. Sauer, and J. Stachel. The origin of gravitational lensing: a postscript to Einstein's 1936 Science paper. Science, 275:184-186, January 1997.

8. J. Surdej and J.-F. Claeskens. Gravitational lens studies with a LMT. 1997. URL ftp://vela. astro.ulg.ac.be/preprints/glslmt.ps.gz

9. J. Surdej, O. Absil, P. Bartczak, E. Borra, J.-P. Chisogne, J.-F. Claeskens, B. Collin, M. De Becker, D. Defrère, S. Denis, C. Flebus, O. Garcet, P. Gloesener, C. Jean, P. Lampens, C. Libbrecht, A. Magette, J. Manfroid, D. Mawet, T. Nakos, N. Ninane, J. Poels, A. Pospieszalska, P. Riaud, P.-G. Sprimont, and J.-P. Swings. The 4m international liquid mirror telescope (ILMT). In Ground-based and Airborne Telescopes. Edited by Stepp, Larry M.. Proceedings of the SPIE, Volume 6267, pp. 626704 (2006), volume 6267 of Presented at the Society of Photo-Optical Instrumentation Engineers (SPIE) Conference, July 2006. doi: 10.1117/12.671695.

10. D. Walsh, R. F. Carswell, and R. J. Weymann. 0957 + 561 A, B - Twin quasistellar objects or gravitational lens. Nature, 279:381-384, May 1979.

11. F. Zwicky. Nebulae as Gravitational Lenses. Physical Review, 51:290-290, February 1937. doi: 10.1103/PhysRev.51.290. 

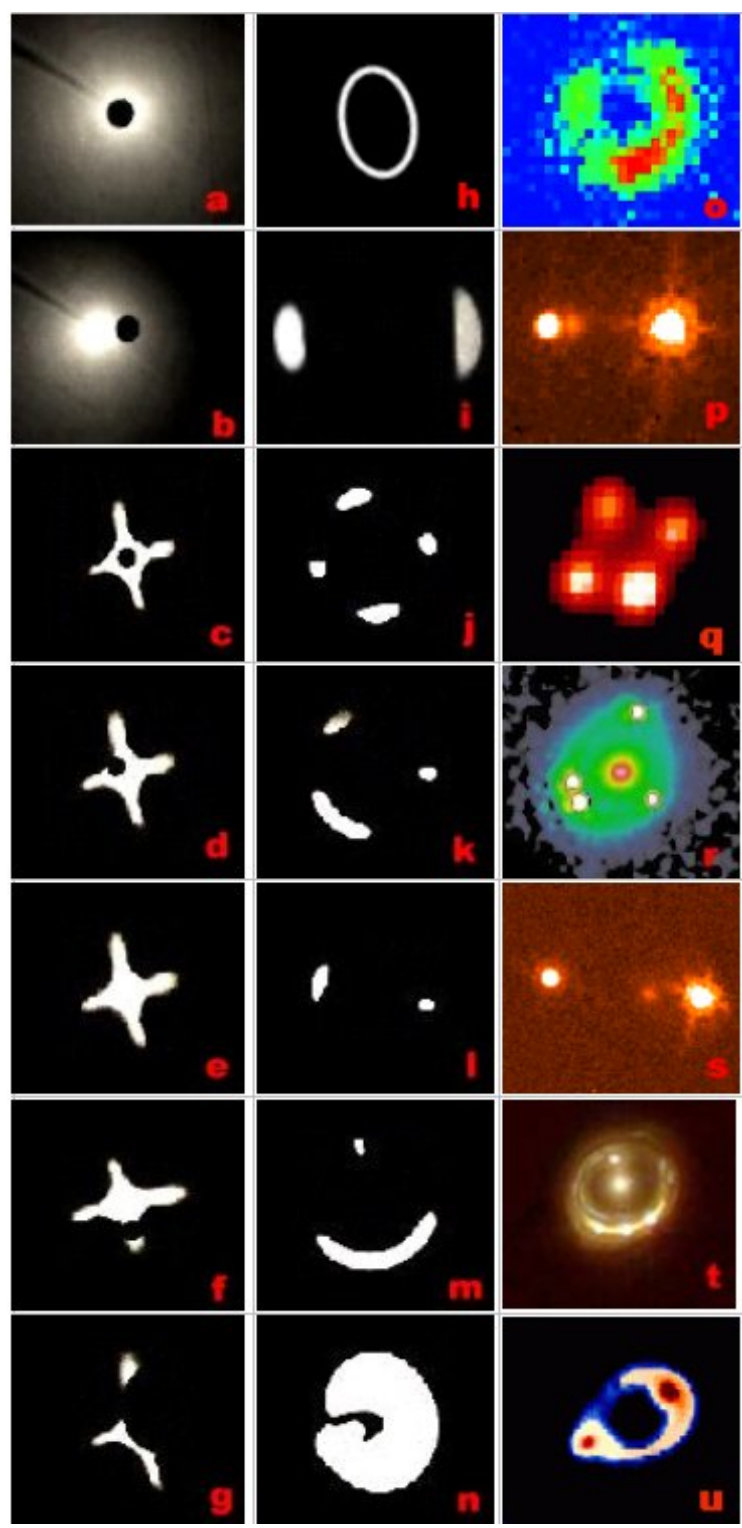

FIGURE 7. Figs. a-g represent the light from a distant source that is redistributed over the pinhole screen by a symmetric (a-b) or an asymmetric (c-g) optical lens and for various positions of the pinhole (observer). Figs. h-n illustrate the corresponding lensed images projected on the large screen located behind the pinhole screen and Figs. o-u display known examples of multiply imaged sources (0047-28078, 1009-0252, H1413+117, PG1115+080, HE1104-1805, RXJ1131-1231 and MG1131+0456). Images (p), (r), (s) and (t) were obtained using the Hubble Space Telescope, the other ones using ground-based facilities (ESO and VLA/NRAO). Courtesy of the European Southern Observatory (ESO), the Space Telescope Science Institute (STScI) operated for NASA by AURA and the Very Large Array (National Radio Astronomy Observatory). 


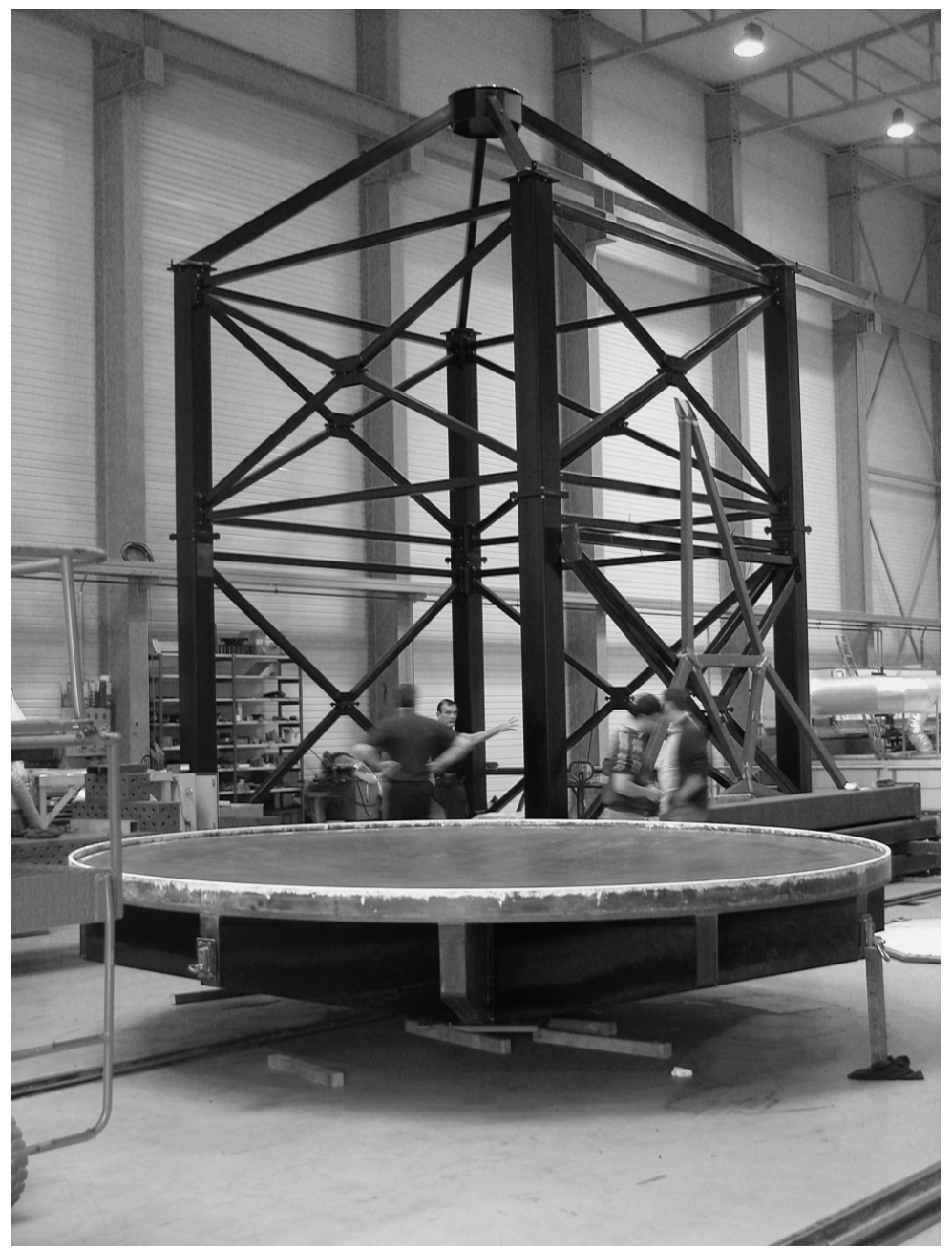

FIGURE 8. The support of the $4 \mathrm{~m}$ liquid mirror made of carbon fiber is seen in the forefront. The structure of the telescope, under construction at AMOS (Liège), is visible just behind. 